\title{
SMM J04135+10277: A CANDIDATE EARLY-STAGE “WET-DRY” MERGER OF TWO MASSIVE GALAXIES AT $z=2.8$
}

\author{
DOMINIK A. RIECHERS ${ }^{1,2}$ \\ ${ }^{1}$ Astronomy Department, California Institute of Technology, MC 249-17, 1200 East California Boulevard, Pasadena, CA 91125, USA \\ ${ }^{2}$ Astronomy Department, Cornell University, 220 Space Sciences Building, Ithaca, NY 14853, USA; dr@ astro.cornell.edu \\ Received 2012 October 4; accepted 2013 February 2; published 2013 February 21
}

\begin{abstract}
We report interferometric imaging of $\mathrm{CO}(J=3 \rightarrow 2)$ emission toward the $z=2.846$ submillimeter-selected galaxy SMM J04135+10277, using the Combined Array for Research in Millimeter-wave Astronomy (CARMA). SMM J04135+10277 was previously thought to be a gas-rich, submillimeter-selected quasar, with the highest molecular gas mass among high- $z$ quasars reported in the literature. Our maps at $\sim 6 \times$ improved linear resolution relative to earlier observations spatially resolve the emission on $\sim 1$ 1".7 scales, corresponding to a (lensing-corrected) source radius of $\sim 5.2 \mathrm{kpc}$. They also reveal that the molecular gas reservoir, and thus, likely the submillimeter emission, is not associated with the host galaxy of the quasar, but with an optically faint gas-rich galaxy at $5^{\prime \prime} .2$, or $41.5 \mathrm{kpc}$ projected distance from the active galactic nucleus (AGN). The obscured gas-rich galaxy has a dynamical mass of $M_{\mathrm{dyn}} \sin ^{2} i=5.6 \times 10^{11} M_{\odot}$, corresponding to a gas mass fraction of $\simeq 21 \%$. Assuming a typical $M_{\mathrm{BH}} / M_{*}$ ratio for $z \gtrsim 2$ quasars, the two galaxies in this system have an approximate mass ratio of $\sim 1$.9. Our findings suggest that this quasar-starburst galaxy pair could represent an early stage of a rare major, gas-rich/gas-poor ("wet-dry") merger of two massive galaxies at $z=2.8$, rather than a single, gas-rich AGN host galaxy. Such systems could play an important role in the early buildup of present-day massive galaxies through a submillimeter-luminous starburst phase, and may remain hidden in larger numbers among rest-frame far-infrared-selected quasar samples at low and high redshift.
\end{abstract}

Key words: cosmology: observations - galaxies: active - galaxies: formation - galaxies: high-redshift galaxies: starburst - radio lines: galaxies

Online-only material: color figures

\section{INTRODUCTION}

Studies of gas- and dust-rich, starbursting active galactic nucleus (AGN) host galaxies out to cosmological distances are important to better understand the connection between supermassive black hole and stellar bulge growth in galaxies that gives rise to the present-day $M_{\mathrm{BH}}-M_{\text {bulge }}$ relation (Magorrian et al. 1998; Häring \& Rix 2004; Graham 2012). A particularly important cosmic epoch for these studies is the redshift range $2 \lesssim z \lesssim 3$ where most of the growth of stellar and black hole mass in galaxies occurs, i.e., where the volume densities of both cosmic star formation and AGN activity peak (e.g., Magnelli et al. 2009; Richards et al. 2006).

It has recently been found that the dynamical masses of some of the most distant quasars at $z>4$ appear to be too small to host stellar components as expected from the local $M_{\mathrm{BH}}-M_{\text {bulge }}$ relation, and that the available gas masses are too small to produce a sufficient amount of stars to approach this relation (e.g., Walter et al. 2004; Riechers et al. 2008a, 2008b). Thus, these galaxies appear to require a source of external gas supply (or stars) to assemble sufficient stellar mass by $z=0$ to approach the local $M_{\mathrm{BH}}-M_{\text {bulge }}$ relation. This gas supply could either be due to accretion of gas through cold streams (e.g., Dekel et al. 2009) or due to gas-rich, gas-rich ("wet-wet") or gas-rich, gaspoor ("wet-dry") mergers with massive and/or gas-rich galaxies (e.g., Springel et al. 2005).

Examples of the latter may be found among high-redshift, submillimeter-selected quasars. A strong submillimeter detection is suggestive of a large amount of warm dust heated by young stars formed at high rates (e.g., Isaak et al. 2002). Followup observations of the molecular interstellar medium (ISM) in these galaxies, typically through the detection of $\mathrm{CO}$ lines, are important to measure the mass of the ISM that constitutes the reservoir for star formation, and to confirm that the starburst and gas are at the same redshift as the AGN (e.g., Coppin et al. 2008).

A particularly interesting submillimeter-selected quasar was found in the field of the $z=0.088$ galaxy cluster Abell 478, SMM J04135+10277 at $z=2.837 \pm 0.003$ (Knudsen et al. 2003). The source was identified in 450 and $850 \mu \mathrm{m}$ observations with the James Clerk Maxwell Telescope/SCUBA instrument, revealing high submillimeter fluxes of $25 \pm 2.8$ and $55 \pm 17 \mathrm{mJy}$, respectively, which suggest a total infrared luminosity of $(2.9 \pm 0.5) \times 10^{13} L_{\odot}$. Subsequent interferometric $\mathrm{CO}(J=3 \rightarrow 2)$ observations at $15^{\prime \prime} \times 11^{\prime \prime}$ resolution and singledish $\mathrm{CO}(J=1 \rightarrow 0)$ observations revealed a massive molecular gas reservoir at $z=2.846 \pm 0.002$, consistent with both the redshift and position of the quasar within the relative uncertainties (Hainline et al. 2004; Riechers et al. 2011a). None of these past studies offered sufficient spatial resolution to spatially resolve and/or precisely locate the $\mathrm{CO}$ or submillimeter continuum emission.

We here report higher spatial resolution $\operatorname{CO}(J=3 \rightarrow 2)$ observations with CARMA to determine the size and dynamical mass of the molecular gas reservoir. We use a concordance, flat $\Lambda \mathrm{CDM}$ cosmology throughout, with $H_{0}=71 \mathrm{~km} \mathrm{~s}^{-1} \mathrm{Mpc}^{-1}$, $\Omega_{\mathrm{M}}=0.27$, and $\Omega_{\Lambda}=0.73$ (Spergel et al. 2003, 2007).

\section{OBSERVATIONS}

We observed the $\operatorname{CO}(J=3 \rightarrow 2)$ transition line $\left(v_{\text {rest }}=\right.$ $345.7959899 \mathrm{GHz}$, redshifted to $89.911 \mathrm{GHz}$, or $3.33 \mathrm{~mm}$ ) 


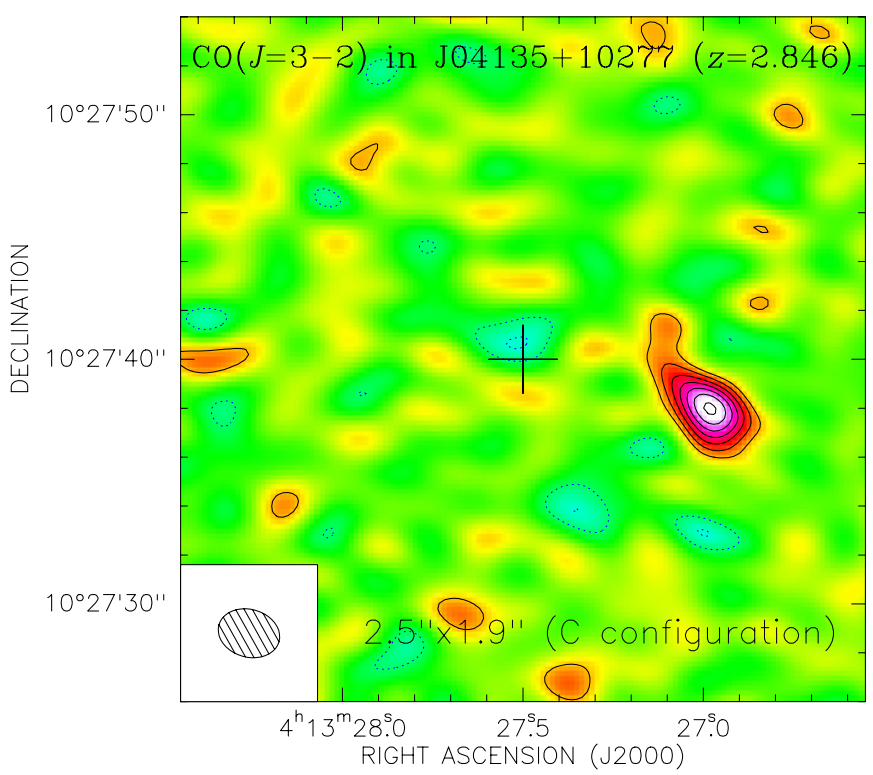

Figure 1. CARMA CO $(J=3 \rightarrow 2)$ map of SMM J04135+10277 over the central $765 \mathrm{~km} \mathrm{~s}^{-1}$. Contours are shown in steps of $1 \sigma=0.555 \mathrm{mJy}^{\text {beam }}{ }^{-1}$, starting at $\pm 2 \sigma$. The cross indicates the pointing center. The synthesized beam size is shown in the lower left corner.

(A color version of this figure is available in the online journal.)

toward SMM J04135+10277, using CARMA. A total bandwidth of $3.7 \mathrm{GHz}\left(\sim 12,400 \mathrm{~km} \mathrm{~s}^{-1}\right.$; at $5.208 \mathrm{MHz}$ resolution) was used to cover the $\mathrm{CO}(J=3 \rightarrow 2)$ line and the underlying $3.33 \mathrm{~mm}$ (rest-frame $870 \mu \mathrm{m}$ ) continuum emission. Observations were carried out under good $3 \mathrm{~mm}$ weather conditions for three tracks in $\mathrm{C}$ configuration (18-367 m baselines, which corresponds to probing spatial scales of $1^{\prime \prime} 5-31^{\prime \prime}$, or $11-250 \mathrm{kpc}$ ) on 2012 February 25, 29, and March 12. This resulted in $6.3 \mathrm{hr}$ of 15 antenna-equivalent on-source time after discarding unusable visibility data. The nearby source $3 \mathrm{C} 120$ was observed every 15 minutes for pointing, amplitude, and phase calibration. Fluxes were bootstrapped relative to Mars. The bright nearby calibrators 3C84 and J0423-013 were observed for bandpass calibration, yielding $\sim 15 \%$ calibration accuracy.

The MIRIAD package was used for data reduction and analysis. All data were mapped using the CLEAN algorithm with "natural" weighting, resulting in a synthesized beam size of 2 ". $5 \times 1$ ". 9 . The final rms is $0.55 \mathrm{mJy}$ beam ${ }^{-1}$ over $229.2 \mathrm{MHz}$ (corresponding to $765 \mathrm{~km} \mathrm{~s}^{-1}$ ), and $1.8 \mathrm{mJy} \mathrm{beam}^{-1}$ over 20.8 $\mathrm{MHz}\left(69 \mathrm{~km} \mathrm{~s}^{-1}\right)$.

\section{RESULTS}

We have detected and spatially resolved strong $\mathrm{CO}(J=3 \rightarrow 2)$ emission toward SMM J04135+10277 (Figure 1). CO emission is detected in each of the tracks individually at the same position. By fitting an elliptical, two-dimensional Gaussian to the $u-v$ data, we find a source size of 1".66 \pm 0.40 along its major axis, corresponding to $13.2 \pm 3.2 \mathrm{kpc}$ at $z=2.846$. The source remains unresolved down to $\lesssim 1^{\prime \prime} .2(\lesssim 9.5 \mathrm{kpc})$ along its minor axis. From fitting a four-parameter Gaussian to the spectrum of the $\mathrm{CO}(J=3 \rightarrow 2)$ line emission (Figure 2), we measure a line peak flux of $6.6 \pm 0.9 \mathrm{mJy}$ at a FWHM velocity width of $679 \pm 120 \mathrm{~km} \mathrm{~s}^{-1}$. Within the relative uncertainties, the line width is consistent with that measured in the $\mathrm{CO}(J=1 \rightarrow 0)$ line $\left(505 \pm 75 \mathrm{~km} \mathrm{~s}^{-1}\right.$; Riechers et al. 2011a), and marginally consistent with a previous measurement of the $\mathrm{CO}(J=3 \rightarrow 2)$ line at lower significance $\left(340 \pm 120 \mathrm{~km} \mathrm{~s}^{-1}\right.$; Hainline et al. 2004). Our measurements correspond to an integrated $\mathrm{CO}(J=3 \rightarrow 2)$ line flux of $4.78 \pm 0.67 \mathrm{Jy} \mathrm{km} \mathrm{s}^{-1}$, and a $\mathrm{CO}(J=3 \rightarrow 2) / \mathrm{CO}(J=1 \rightarrow 0)$ brightness temperature ratio of $r_{31}=0.82 \pm 0.15$. The Gaussian peaks at a redshift of $z_{\mathrm{CO}}=$ $2.8458 \pm 0.0006$, consistent with previous estimates within the errors. We marginally detect the underlying $3.33 \mathrm{~mm}$ continuum emission at a level of $0.31 \pm 0.17 \mathrm{mJy}$.

The $\operatorname{CO}(J=3 \rightarrow 2)$ emission peaks at a position of $\alpha=$ $04^{\mathrm{h}} 13^{\mathrm{m}} 26^{\mathrm{s}} .989 \pm 0^{\prime \prime} .11, \delta=+10^{\circ} 27^{\prime} 37^{\prime \prime} .89 \pm 0^{\prime \prime} .12$. The $i$-band position of the quasar as determined from a Hubble Space Telescope WFPC2 F814W image obtained from the Hubble Legacy Archive ${ }^{3}$ is $\alpha=04^{\mathrm{h}} 13^{\mathrm{m}} 27^{\mathrm{s}} .28, \delta=+10^{\circ} 27^{\prime} 40^{\prime \prime} .77$. Thus, the molecular gas reservoir is spatially offset by $5^{\prime \prime} .2$, or $41.5 \mathrm{kpc}$, from the AGN position (Figure 3). There is no evidence for any rest-frame $\sim 210 \mathrm{~nm}$ emission at the position of the CO emission. Spitzer Space Telescope IRAC 3.6-8.0 $\mu \mathrm{m}$ images obtained from the Spitzer Heritage Archive ${ }^{4}$ reveal a

\footnotetext{
3 http://hla.stsci.edu

4 http://sha.ipac.caltech.edu
}

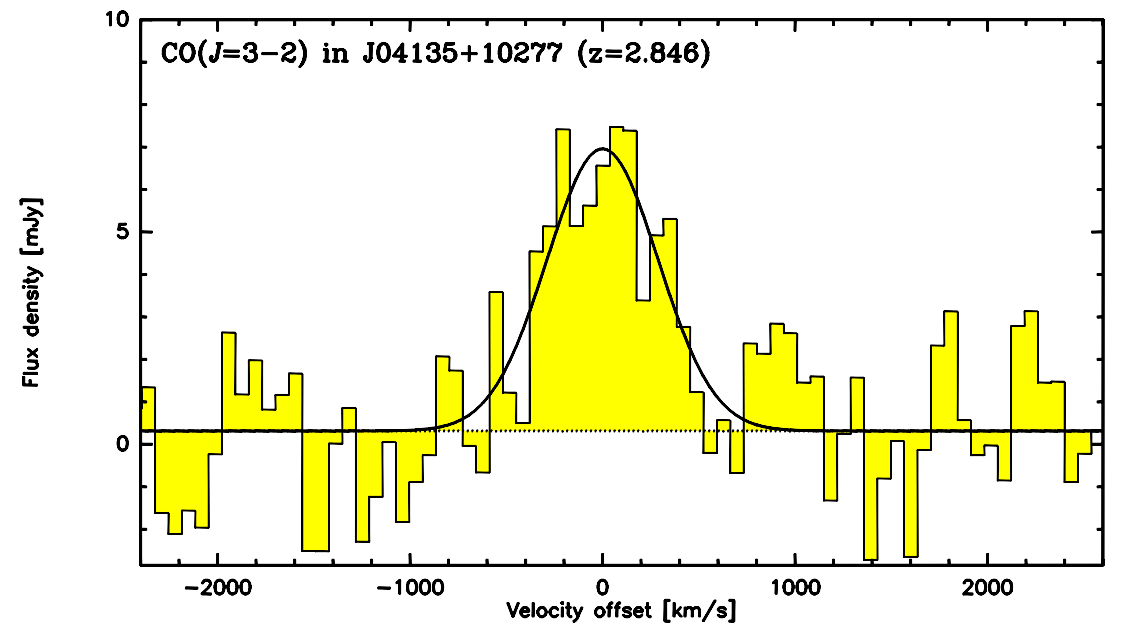

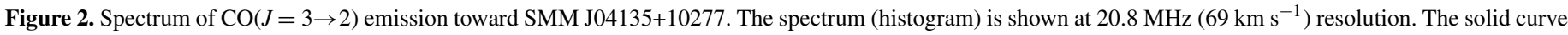
indicates a Gaussian fit to the spectrum.

(A color version of this figure is available in the online journal.) 


\section{SMM J04135+10277}

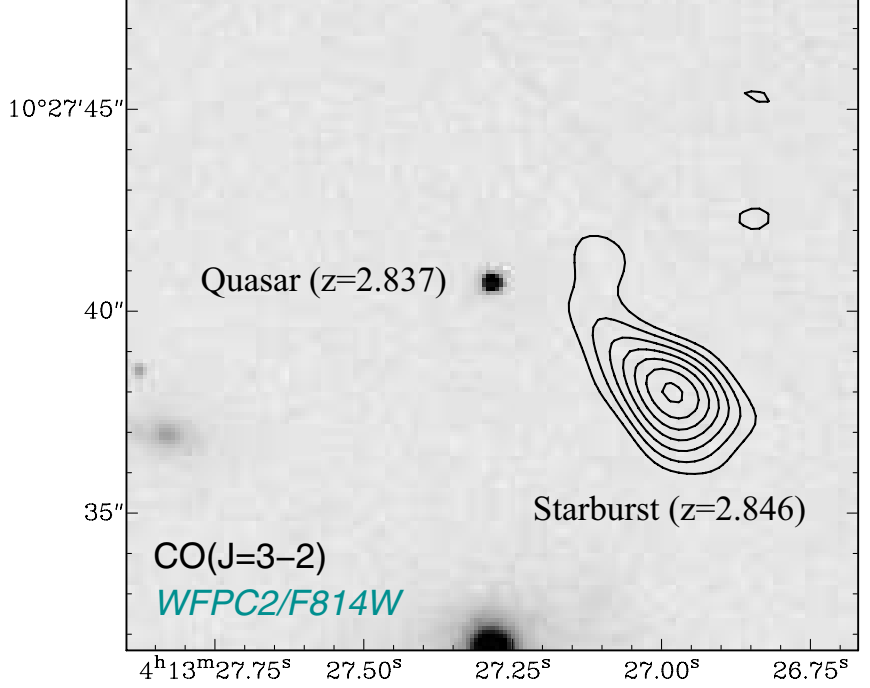

Figure 3. Overlay of $\mathrm{CO}(J=3 \rightarrow 2)$ emission (contours) toward SMM J04135+10277 on a Hubble Space Telescope WFPC2 F814W image (rest frame $\sim 210 \mathrm{~nm}$ ). The $\mathrm{CO}$ emission is spatially offset by $5^{\prime \prime} .2$ from the position of the optical quasar. No optical emission is detected at the position of the $\mathrm{CO}$ emission.

(A color version of this figure is available in the online journal.)

faint counterpart to the $\mathrm{CO}$-emitting galaxy at rest-frame nearinfrared wavelengths $(0.9-2.1 \mu \mathrm{m}$; Figure 4). Its rest-frame near-infrared spectrum appears to be flatter than that of the quasar, consistent with a dust-obscured star-forming galaxy and the lack of a strong AGN component. There is no evidence for any $\mathrm{CO}$ or continuum emission at the position of the quasar. Assuming a line FWHM of $400 \mathrm{~km} \mathrm{~s}^{-1}$, we derive a $3 \sigma$ upper limit of $0.9 \mathrm{Jy} \mathrm{km} \mathrm{s}^{-1}$ for the $\mathrm{CO}(J=3 \rightarrow 2)$ line flux of the quasar host galaxy. This corresponds to $<20 \%$ of the $\mathrm{CO}(J=3 \rightarrow 2)$ line flux of the submillimeter source. We also derive a $3 \sigma$ upper limit of $0.4 \mathrm{mJy}$ for the $3.33 \mathrm{~mm}$ continuum emission at the position of the quasar.

\section{ANALYSIS}

To account for a small amount of gravitational magnification by the foreground galaxy cluster, we will adopt a lensing magnification factor of $\mu_{\mathrm{L}}^{\mathrm{QSO}}=1.3$ for the AGN (as determined by Knudsen et al. 2003), and of $\mu_{\mathrm{L}}^{\mathrm{CO}}=1.6 \pm 0.5$ for the molecular gas and far-infrared continuum emission (as determined using the $\mathrm{CO} J=1 \rightarrow 0$ line luminosity and FWHM from Riechers et al. 2011a and a $\mu_{\mathrm{L}}-L_{\mathrm{CO}(1-0)}^{\prime}-d v_{\mathrm{FWHM}}$ scaling relation for submillimeter-selected galaxies; Harris et al. 2012) in the following. We further adopt the gas mass determined by Riechers et al. (2011a) based on the $\operatorname{CO}(J=1 \rightarrow 0)$ line luminosity, but scaled to our updated lensing magnification factor. This suggests $M\left(\mathrm{H}_{2}\right)=1.2 \times 10^{11}\left(1.6 / \mu_{\mathrm{L}}\right)^{-1} M_{\odot}$ (assuming a $L_{\mathrm{CO}(1-0)}^{\prime}-M\left(\mathrm{H}_{2}\right)$ conversion factor of $\alpha_{\mathrm{CO}}=$ $0.8 M_{\odot}\left(\mathrm{K} \mathrm{km} \mathrm{s}^{-1} \mathrm{pc}^{2}\right)^{-1}$ for ultra-luminous infrared galaxies (ULIRGs); Downes \& Solomon 1998, but also see recent discussion by Papadopoulos et al. 2012). The size estimate (5.2 kpc radius; corrected by a factor of $\mu_{\mathrm{L}}^{1 / 2}$; e.g., Riechers et al. 2009) and width of the CO line suggest a dynamical mass of $M_{\mathrm{dyn}} \sin ^{2} i=5.6 \times 10^{11} M_{\odot} \cdot{ }^{5}$ This suggests a gas mass fraction of $f_{\text {gas }}=M\left(\mathrm{H}_{2}\right) /\left(M_{\mathrm{dyn}} \sin ^{2} i\right) \simeq 21 \%$.

To determine the black hole mass of the quasar, we adopt the $\lambda L_{5100 \AA}$ luminosity determined by Knudsen et al. (2003) and the $M_{\mathrm{BH}}-L_{5100 \AA}$ relationship determined by Peterson et al. (2004). This suggests a black hole mass of $M_{\mathrm{BH}}=1.7 \times 10^{9} M_{\odot}$. If the source were to follow the $M_{\mathrm{BH}}-M_{\text {bulge }}$ relation for nearby galaxies (Häring \& Rix 2004), this would suggest $M_{\text {bulge }} \simeq$ $8.2 \times 10^{11} M_{\odot}$. However, Peng et al. (2006) suggest that this ratio is likely typically $\sim 4 \times$ lower at $z \sim 2$. In the following, we thus assume a stellar mass of $M_{\star} \simeq 2 \times 10^{11} M_{\odot}$ for the quasar host galaxy. Approximating the total mass of the system to be $M_{\text {tot }}=M_{\mathrm{BH}}+M_{\star}+M\left(\mathrm{H}_{2}\right)+M_{\text {dust }}+M_{\mathrm{DM}} \simeq 3 \times 10^{11} M_{\odot}$ (adopting a gas-to-dust ratio of 100, and assuming a contribution from dark matter (DM) of 25\%), we find a gas fraction of $f_{\text {gas }}^{\mathrm{t}}=M\left(\mathrm{H}_{2}\right) / M_{\mathrm{tot}}<8 \%$ (assuming the same gas excitation as for the $\mathrm{CO}$-detected galaxy).

\section{DISCUSSION AND CONCLUSIONS}

We have imaged $\mathrm{CO}(J=3 \rightarrow 2)$ emission toward the highredshift galaxy SMM J04135+10277, using CARMA. Our observations suggest that the molecular gas reservoir previously detected toward SMM J04135+10277 is not associated with the

\footnotetext{
5 Due to the flattening of the baryonic mass distribution in a disk, possible biases due to clumpiness of the gas, and non-circular motions of the gas, virial estimates for clumpy, disk-like galaxies may underpredict the dynamical mass by typically $\sim 30 \%$ (e.g., Daddi et al. 2010). Estimates for more complex dynamical systems likely exhibit at least comparable uncertainties, and thus, require model-based correction factors. For submillimeter-bright dusty starburst galaxies, an isotropic virial estimator is commonly adopted, which gives by a factor of $\sim 1.5$ larger values than standard estimates for rotating disk galaxies at an average inclination (Engel et al. 2010). Adopting the isotropic virial estimator instead of the inclined disk model used here would result in a $\sim 20 \%$ larger dynamical mass relative to an edge-on configuration. Both estimators result in the same value when assuming an inclination of $i \simeq 66^{\circ}$. The uncertainties on the dynamical mass estimate, and thus, $f_{\text {gas }}$, amount to at least $20 \%-30 \%$
}

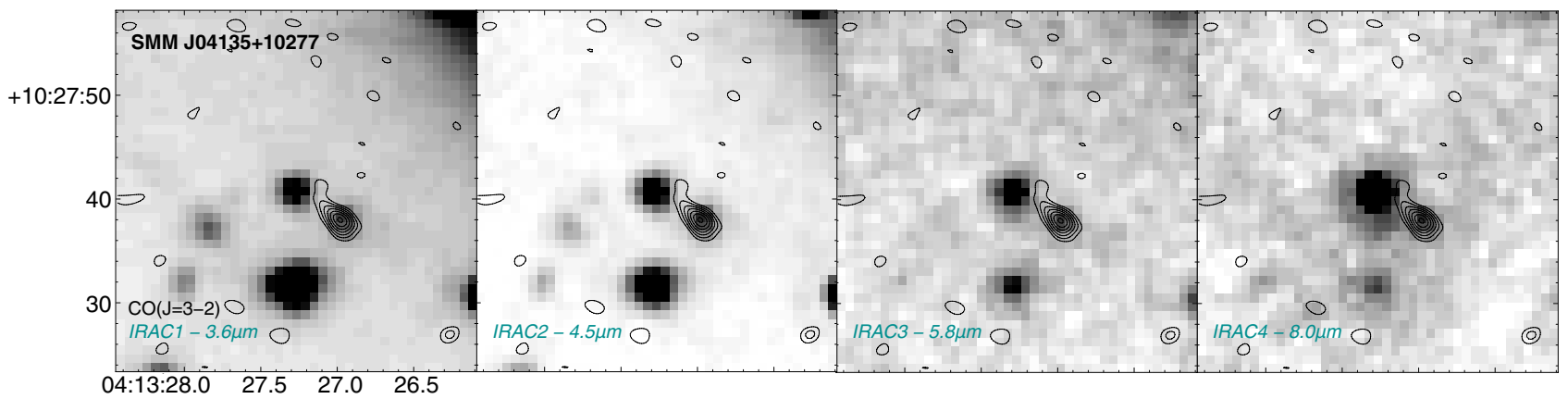

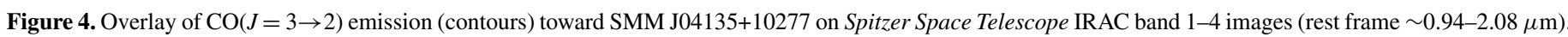
Faint emission is detected at the position of the $\mathrm{CO}$ emission in all IRAC bands, with a spectral slope flatter than that of the optical quasar.

(A color version of this figure is available in the online journal.) 
host galaxy of the optically detected quasar at $z=2.837$, but with an optically faint, gas-rich galaxy at $z=2.846$, separated by $\gtrsim 40 \mathrm{kpc}$ from the AGN. The spectral properties of the CO-emitting source are consistent with those of an optically obscured star-forming galaxy. Its $\mathrm{CO}(J=3 \rightarrow 2) / \mathrm{CO}(J=1 \rightarrow 0)$ brightness temperature ratio of $r_{31}=0.82 \pm 0.15$ is within the range of values observed for $z>2$ submillimeter-selected starburst galaxies (e.g., Riechers et al. 2011b, 2011c; Ivison et al. 2011), but higher than the values typically observed in high- $z$ disk galaxies (e.g., Dannerbauer et al. 2009; Aravena et al. 2010). Assuming no difference in CO excitation, this gas-rich companion carries at least $5 \times$ the gas mass of the quasar host galaxy. ${ }^{6}$ The gas mass and gas mass fraction of the companion are comparable to those of other $z>2$ submillimeter galaxies (e.g., Tacconi et al. 2006; Riechers et al. 2011d). We estimate the total mass of the SMM J04135+10277 system to be $\gtrsim 8.6 \times 10^{11} M_{\odot}$ (with considerable uncertainty), which may be dominated by the gas-rich companion galaxy (mass ratio of $\sim 1.9$ ).

Gas-rich companions have been detected in other high$z$ quasars as well, such as the $z=4.4$ and 4.7 systems BRI 1335-0417 (which is already actively merging with its companion on $5 \mathrm{kpc}$ scales; Riechers et al. 2008b) and BR 1202-0725 (which has a companion at $26 \mathrm{kpc}$ projection; e.g., Carilli et al. 2002, 2013). However, in all examples known so far at high- $z$, both the quasar host and companion are gas-rich. Thus, the quasar-starburst galaxy pair SMM J04135+10277 could be the first high-redshift example of an early-stage gasrich, gas-poor merger, in which the optically faint submillimeter galaxy provides the gas supply to further build up the stellar component of the quasar host.

Models of hierarchical structure formation (e.g., Springel et al. 2005) lend support to the idea that a close massive galaxy pair like SMM J04135+10277 will likely result in a major merger in which the gas-rich companion may replenish the gas supply in the quasar host (which perhaps will yield configurations similar to those observed in nearby infrared-luminous galaxies in intermediate or late merger stages that contain both AGN and gas-rich galaxy components; e.g., Evans et al. 2002). The relatively large projected separation of $\gtrsim 40 \mathrm{kpc}$ suggests that the two massive galaxies are likely physically related and gravitationally interacting, but cannot (yet) be considered part of a common gravitational potential. This is consistent with what is expected for a merging system in an early stage. Given the early phase in the merging process implied by this scenario, it is plausible but not unambiguous to assume that the ongoing black hole accretion in the quasar and star formation in the gas-rich companion could have been triggered by interaction.

Submillimeter-selected high-redshift quasars are good candidates for transition objects from hyper-luminous infrared galaxies to optically bright quasars, linking the most intense starbursts in the universe to the most actively accreting black holes (e.g., Coppin et al. 2008; Simpson et al. 2012). This scenario would be consistent with a high-redshift analog of the ULIRG-quasar transition scenario proposed by Sanders et al. (1988). However, despite being a good candidate for a transition object initially, SMM J04135+10277 is not an example of such sources. Instead, our observations suggest that it is a good candidate for

\footnotetext{
6 High-redshift quasar host galaxies commonly show high $r_{31}$ of $>0.9$, which may suggest an even higher ratio in gas mass (e.g., Riechers et al. 2006, 2011a).
}

an early-stage gas-rich, gas-poor ("wet-dry") merger" of two massive galaxies at $z=2.8$, and thus, a possibly more extreme high-redshift analog to the $z=0.3$ quasars HE 0450-2958 and $\mathrm{J} 1821+643$ (which were identified to not be transition objects through similar observational strategies; e.g., Papadopoulos et al. 2008; Aravena et al. 2011).

Gas-rich, gas-poor ("wet-dry") mergers at high redshift are predicted by cosmological simulations of hierarchical structure formation (e.g., Springel et al. 2005), but successful observations of such systems are still scarce. Based on the discovery of SMM J04135+10277 alone, it remains unclear what the incidence of such systems within submillimeter-selected quasar samples is. It however clearly motivates observations of larger samples with high-quality optical/infrared data in CO and submillimeter continuum emission at high spatial resolution. Such studies will become feasible in the near future, with the completion of both the Karl G. Jansky Very Large Array (VLA) and the Atacama Large (sub-) Millimeter Array (ALMA).

We thank the anonymous referee for a helpful report. D.R. acknowledges support from the National Aeronautics and Space Administration (NASA) through a Spitzer Space Telescope grant. Support for CARMA construction was derived from the G. and B. Moore Foundation, the K. T. and E. L. Norris Foundation, the Associates of the California Institute of Technology, the states of California, Illinois, and Maryland, and the NSF. Ongoing CARMA development and operations are supported by the NSF under a cooperative agreement, and by the CARMA partner universities. Based in part on observations made with the NASA/ESA Hubble Space Telescope, and obtained from the Hubble Legacy Archive, which is a collaboration between the Space Telescope Science Institute (STScI/NASA), the Space Telescope European Coordinating Facility (ST-ECF/ESA), and the Canadian Astronomy Data Centre (CADC/NRC/CSA). Based in part on observations made with the Spitzer Space Telescope, and obtained from the Spitzer Heritage Archive through the NASA/IPAC Infrared Science Archive, which is operated by the Jet Propulsion Laboratory, California Institute of Technology, under contract with NASA.

\section{REFERENCES}

Aravena, M., Carilli, C., Daddi, E., et al. 2010, ApJ, 718, 177

Aravena, M., Wagg, J., Papadopoulos, P. P., \& Feain, I. J. 2011, ApJ, 737, 64

Carilli, C. L., Kohno, K., Kawabe, R., et al. 2002, AJ, 123, 1838

Carilli, C. L., Riechers, D., Walter, F., et al. 2013, ApJ, 763, 120

Coppin, K., Swinbank, A. M., Neri, R., et al. 2008, MNRAS, 389, 45

Daddi, E., Bournaud, F., Walter, F., et al. 2010, ApJ, 713, 686

Dannerbauer, H., Daddi, E., Riechers, D. A., et al. 2009, ApJL, 698, L178

Dekel, A., Birnboim, Y., Engel, G., et al. 2009, Natur, 457, 451

Downes, D., \& Solomon, P. M. 1998, ApJ, 507, 615

Engel, H., Tacconi, L. J., Davies, R. I., et al. 2010, ApJ, 724, 233

Evans, A. S., Mazzarella, J. M., Surace, J. A., \& Sanders, D. B. 2002, ApJ, 580,749

Graham, A. W. 2012, ApJ, 746, 113

Hainline, L. J., Scoville, N. Z., Yun, M. S., et al. 2004, ApJ, 609, 61

Häring, N., \& Rix, H.-W. 2004, ApJL, 604, L89

Harris, A. I., Baker, A. J., Frayer, D. T., et al. 2012, ApJ, 752, 152 Isaak, K. G., Priddey, R. S., McMahon, R. G., et al. 2002, MNRAS, 329, 149 Ivison, R. J., Papadopoulos, P. P., Smail, I., et al. 2011, MNRAS, 412, 1913 Knudsen, K. K., van der Werf, P. P., \& Jaffe, W. 2003, A\&A, 411, 343 Magnelli, B., Elbaz, D., Chary, R. R., et al. 2009, A\&A, 496, 57

\footnotetext{
7 We note that "dry" here refers to the fact that the companion galaxy far dominates the gas content of this system. It does not imply that there is no gas whatsoever in the host of the quasar.
} 
Magorrian, J., Tremaine, S., Richstone, D., et al. 1998, AJ, 115, 2285

Papadopoulos, P. P., Feain, I. J., Wagg, J., \& Wilner, D. J. 2008, ApJ, 684,845

Papadopoulos, P. P., van der Werf, P., Xilouris, E., Isaak, K. G., \& Gao, Y. 2012, ApJ, 751, 10

Peng, C. Y., Impey, C. D., Rix, H.-W., et al. 2006, ApJ, 649, 616

Peterson, B. M., Ferrarese, L., Gilbert, K. M., et al. 2004, ApJ, 613, 682

Richards, G. T., Strauss, M. A., Fan, X., et al. 2006, AJ, 131, 2766

Riechers, D. A., Carilli, C. L., Maddalena, R. J., et al. 2011a, ApJL, 739, L32

Riechers, D. A., Carilli, C. L., Walter, F., et al. 2011b, ApJL, 733, L11

Riechers, D. A., Cooray, A., Omont, A., et al. 2011c, ApJL, 733, L12

Riechers, D. A., Hodge, J., Walter, F., Carilli, C. L., \& Bertoldi, F. 2011d, ApJL, 739, L31
Riechers, D. A., Walter, F., Brewer, B. J., et al. 2008a, ApJ, 686, 851

Riechers, D. A., Walter, F., Carilli, C. L., Bertoldi, F., \& Momjian, E. 2008b, ApJL, 686, L9

Riechers, D. A., Walter, F., Carilli, C. L., \& Lewis, G. F. 2009, ApJ, 690,463

Riechers, D. A., Walter, F., Carilli, C. L., et al. 2006, ApJ, 650, 604

Sanders, D. B., Soifer, B. T., Elias, J. H., et al. 1988, ApJ, 325, 74

Simpson, J., Smail, Ian, Swinbank, A. M., et al. 2012, MNRAS, 426, 3201

Spergel, D. N., Bean, R., Doré, O., et al. 2007, ApJS, 170, 377

Spergel, D. N., Verde, L., Peiris, H. V., et al. 2003, ApJS, 148, 175

Springel, V., White, S. D. M., Jenkins, A., et al. 2005, Natur, 435, 629

Tacconi, L. J., Neri, R., Chapman, S. C., et al. 2006, ApJ, 640, 228

Walter, F., Carilli, C., Bertoldi, F., et al. 2004, ApJL, 615, L17 